\title{
PRESUPPOSTI STORICO-LETTERARI ED ESTETICI DEL ROMANZO STORICO
}

\section{Leopoldo MELANI}

Poiché il romanzo storico é una forma letteraria caratteristica del periodo romantico, per comprenderne l'origine e i caratteri, é nесевваrio richiamare brevemente alla memoria le peculiarità essenfiall del Romanticismo al suo inizio e nei suoi sviluppi.

Ocorre inoltre tener presente che il Romanticismo italiano, sebhene s'inquadri nel movimento generale europeo di origine germaHea, ha caratteri propri e inconfondibili, talvolta $c^{\sim}$ ntrastanti con il llomantic'smo straniero. A questo proposito gioverà osservare che Hililia aveva avuto dei precursori nel '700, come il Vico, che già aveva intuito il carattere sentimentale e fantastico della poesia; come II Baretti che aveva combattuto, tra l'altro, le unità aristoteliche il dileso Shakespeare; come il Cesarotti che aveva tradotto Ossian.

Inoltre l'Alfieri prima e il Foscolo poi, il Monti e il Pindemonle, acbbene classicisti, in certune delle loro opere avevano manifesiato caratteri del tutto moderni.

Quanto ai concetti moralistici dell'arte, si può parlare di un allinamento dell'opera pariniana.

II movimento straniero offrí lo spunto, la mossa iniziale; e il va fu dato, come si sa, dalla traduzione del libro "De l'Alemagne" della Stael (1814) e dalla "Lettera semiseria di Grisostomo" del Herchet (1816).

HowA Per questo anggio di si é valsi specialmente degli studi di Umberto 1Haice (E) 1. Mararali, 1040) a di Mario Praz (Rapporti tra la letteratura italiana e la letInitin inisias, in Letterature comparate, Marzorati, 1948) ambedue pubblicati IIi collana "Froblemi ed orientamenti critici di lingua e letteratura italiana»,

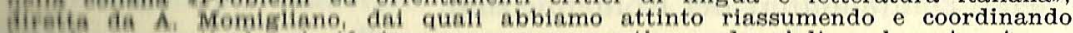
(H)i afumenti the diferivane per una questione od un'altra al nostro tema.

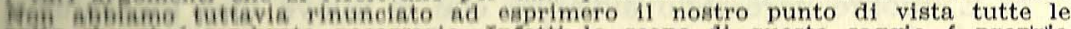

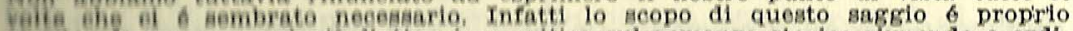

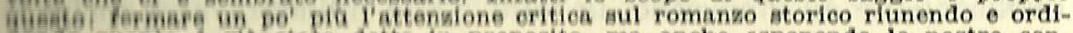

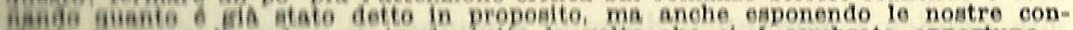

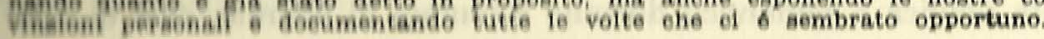


Cerchiamo quindi di richiamare alla memoria | caratteri elementari, per cosí dire, e fondamentali della prima letteratura romantica, ispirati, oltre che al Vico e alla Stael, anche allo Schlegel e al Sismondi:

1) L'arte deve essere popolare, basata sulla natura e sul vero per trovare adesione presso tutte le classi sociali. Non deve quindi ispirarsi ai modelli classici, accademici, né, tanto meno, alla mitologia.

Deve ispirarsi a motivi e sentimenti reali, moderni e morali, come ad esempio, la religione cristiana e la storia medioevale da cui traggono origine le attuali condizioni storiche dei popoli.

2) L'arte deve propcrsi un fine morale per migliorare i costumi Sono insomma quegli ideali contenuti nella nota formula manzoniana:

"La poesia e la letteratura in genere deve proporsi l'utile per iscopo, il vero per soggetto, l'intenessante per mezzo".

Contro il moderno ideale persisteva però l'idea classicistica . Petanto i due periodici "Biblioteca italiana" e il "Conciliatore" soatenitore delle nuove idee romantiche polemizzavano vivamente.

Classico é colui che sente la vita come continuità, che sente cioé di vivere nello stesso clima e sotto lo stesso cielo degli uomini e deili acrittori antichi. Certamente però non possono appagare oggi gli ideali del passato e forse del futuro, se non appagano anche nel presente.

Romantico é, al contrario, colui al quale il passato appare come cosa diversa dall'oggi e il domani un mistero. Non esistono valoHi etemi, perenni, leggi, e la vita é un continuo divenire. Il mondo ha inizio dai singoli individui $\mathrm{e}$ in ogni individuo in ogni diverso instante. Questo é il nucleo, la spinta, il motore della spiritualità romantica, da cui scaturiscono i caratteri più vari ed apparentemente contraddittori del Romanticismo.

$E^{\prime}$ inoltre necessario avvertire che le nuove idee romantiche, ivoluzionarie ed innovatrici in fatto d'arte influenzavano anche la vita politica e romantico divenne sinonimo di liberale, cioé di anti- conformista e rinnovatore, tanto che l'Austria soppresse il Conciliatore.

L'ardore della libertà era stato acceso dalla Rivoluzione francese e l'idealismo trascendente, diffusosi con il Romanticismo, che faceva cercare la felicità nel Divino, nell'Infinito e nell'Eterno, ispirava quel senso di scontentezza a causa dell'irraggiungibilità di questo ideale, producendo le famose crisi romantiche che giungevano perfino alla pazzia e al suicidio.

Il senso della storia si era poi acceso ed acuito con la crisi della Rivoluzione francese stessa.

Ma tutto questo é semplicistico e schematicamente didattico. Infatti ogni movimento e ogni scuola letteraria (se si voglia considerare il Romanticismo come una semplice scuola letteraria) ha radici nella storia del momento e presenta caratteri già visibili fino dalle antiche letterature, anche se non ancora codificati e fatto assurgere a vera e propria norma teorica.

Nasce da particolari condizioni sociali e politiche, da determinate condizioni e orientamenti del pensiero e del gusto. Cosicché le poetiche delle scuole letterarie sono l'aspetto appariscente di una ben più profonda causa e di un ben più profondo atteggiamento spirituale. Da ciò deriva il contrasto fra i dettami della scuola e gli atteggiamenti dello spirito di alcuni scrittori che pure a quella scuola si ispirano. Perché altra cosa é la scuola e altra cosa é lo spirito di un movimento.

Nel caso del Romanticismo potremo citare Leopardi e Foscolo, nemici dichiarati del moderno movimento inteso come dettame di canoni letterari, ma prettamente romantici per certi atteggiamenti del loro spirito. Il contrario succede nel Manzoni, aderente in pieno alle teorie e poetiche del tempo, anzi capo della teoria egli stesso, che invece per certi atteggiamenti del suo spirito é intimamente un classico.

L'arte nel Romanticismo é completa autonomia e libertà individuale, sebbene si trovi in contrasto quando afferma che devono essere perseguiti ideali morali e specialmente politici.

Bisogna poi osservare che se per Romanticismo s'intende il devenire e per classico l'essere, oppure per romantico impeto e per classico equilibrio, ci accorgiamo che questa concezione é di ogni tempo e di ogni paese. 
Ma a noi interessa vedere ciò che accade nel Romanticismo sto. Heo, quando cioé il Romanticismo, per così dire perenne (secondo Ia delinizione di U. Bosco), visibile in tutti i tempi, diventa consapevale ed acquista consistenza storica, si concreta in forme letterarie, diventa reale e quindi classico, come direbbe il Croce, detta precise nerme letterarie.

I Romantici si opposero ai classici che erano, si può dire, gli Illuministi: ma bisogna star bene attenti al significato vero di questa apposizione, sia perché l'llluminismo é pervaso di sentimenti romanili, e ei non soltanto per la perennità di essi, ma anche perché 6eitn movimento é sempre la trasformazione continuatrice del prefedente.

Tuttavia nell'età romantica lo storicismo e l'idealismo si fanno

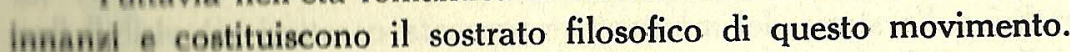
Alla illimitata fiducia nella ragione e nelle verità eterne ed immutabili, al oppone un irriducibile individualismo ed un altrettanto irridueibile nazionalismo.

Tulto acquista un carattere problematico e relativistico (Coneetii basilari, questi, su cui si fonda il pensiero più moderno dei noait giomi).

Se arte 6 espressione individuale, non devono esistere poetilis, canoni prestabiliti, neppure, a rigore, una poetica romantica. It) aueato senso va anche intesa l'abolizione delle unità aristoteliche, delle imitazioni di qualsiasi natura e specialmente della mitologia. Aite é vera vita vissuta, ispirazione reale e sentita. Il che, se bene if analizza, costituisce una vera e propria poetica, fatto che i Romantiel volevano evitare. (1)

E' di questo tempo anche il sorgere dell'antitesi fra "poesia" "letteratura". Poesia é qualcosa di primigenio, istintivo, intimo, die aloore da qualsiasi mezzo letterario di espressione. E' qualcosa hie ai aente e non si esprime. Volendo esprimerla, si sofistica, si allera e si retoricizza. (Fondamento, questo, di tante estetiche recenti he trova nel Romanticismo la sua origine).

Ma l'espressione letteraria é tuttavia necessaria. Perciò tanto piô frande sard il poeta colto, quanto più si avvicinerà alla psico(1) Malie apease infatt, quando l'uomo crede di abolire un determinato fatto, mracacente. logia primigenia ed aurorale del popolo, quanto più dimenticherà la letteratura.

Dice U. Bosco (op. cit. pag. 604) : "Nasce così la distinzione tra concetto di "poesia" e quello di "letteratura", sin qui costituenti un concetto unico; distinzione che con tempo diverrà opposizione. "Letteratura" é ogni diaframma tradizionalistico tra ispirazione ed espressione; la poesia é concepita come una condizione aurorale, primigenia dello spirito, alla quale si contrappone, ostacolandone l'espressione, contaminandone l'essenziale purezza, tutto il bagaglio mitico, linguistico, tecnico-stilistico, che costituisce appunto la "letteratura". Il poeta ideale é colui che scrive come "ditta dentro"; ogni intuizione di origine raziocinativa e culturale turba l'interno dettato, nel quale solo consiste la poesia. Si pensi alla tipicamente romantica concezicne, nel Leopardi, della poesia come pura lirica; la sua riserva circa il Parini, il Monti, in un secondo tempo perfino del Petrarca, ecc. Nasce il grande mito del secolo romantico: quello della poesia ingenua, antiletteraria, che sarà portato dal decadentismo alle sue estreme conseguenze: e correlativamente, il mito della assoluta "immediatezza" espressiva. Solo in tempi recentissimi si é andata e si va sempre più rafforzando la coscienza che non esistono sentimenti "poetici" in sé, astratti, da poter considerare separatamente dalla loro concreta realizzazione nella pagina: che dunque non solo il diaframma non é eliminabile, ma é necessario; che una voce poetica, per essere udita, per diventare cioé messaggio di pcesia valevole per tutti, deve necessariamente essere filtrata attraverso un mezzo "letterario", attraverso regole, sia pure stabilite dallo stesso poeta nell'atto della sua creazione. C'é sempre un tanto, poco o molto, di convenzionale, ineliminabile, nel quale poeta e lettore debbono consentire; sia che quelle regole il lettore le conosca ab initio, per via retorica, sia che le scopra nel momento di leggere il suo poeta".

Si devono indagare e meditare i tesori della psicologia popolare, il folklore, la tradizione popolare (Sono di questo tempo gli studi sulle leggende medioevali). Poesia deve essere interpretazione, esaltazione e guida dei sentimenti popolari e patriottici. Per questo motivo fu tanto in voga la poesia bardita e a questo proposito occorre anche osservare come già nelle premesse del Romanticismo appala l'aspetto finalistico di guida morale e quindi politica del movi- 
mento; ciò che per l'Italia vorrà dire formazione dell'idea liberale di indipendenza, presupposti del Risorgimento.

Si noti pure l'evidente contraddizione tra l'aspetto individuale lell'arte e quello finalistico che essa si propone; contraddizione che divenne poi cosciente anche negli stessi Romantici: si avvertiva il contrasto tra il fine patriottico della loro poetica con la libera espanlone dell'individualità del poeta e l'autonomia dell'arte.

L'altra esigenza caratteristica dell'arte romantica é quella dello abbandono al sogno, all'immediatezza della sensazione, senza preoccuparione letteraria, ccme già abbiamo detto.

Poesia é qualcosa che si sente e non si esprime. Il silenzio é il vero rappresentante della suggestione poetica. Pensieri da cui scaturianno poi movimenti poetici recenti e contemporanei: simbolimo, misticismo, decadentismo, ecc.

II romantico, ben lontano ancora dagli estremismi dei movimenti ricordati, sente tuttavia l'inadegualezza dell'espressione, il contrasto fra ciò che sente e ciò che esprime ed é portato amaramente all'ironia di se stesso.

C'é dunque un'insoddisfazione della parola e la tendenza veran la pagina bianca e verso la risoluzione della parola in musica: una vera arte dell'ineffabile.

Si giungerà alla poesia-musica, alla parola intesa come puro aหono, segno universale di suscitamento di sentimenti, sensazioni e atati d'animo. (In questo, credo, il Pascoli é il vero e più genuino discendente ed erede del movimento romantico). E il sentimentaliamo romantico, tanto bistrattato e deriso, se inteso in questo senso E da caso causato, si presenta sotto un aspetto ben diverso ed ha radiel molto differenti da quelle credute.

II Romanticismo presenta così due altri aspetti tra loro conirastanti: l'aspirazione alla massima concretezza dell'arte, la cui ispirazione deve affondare le radici nella realtà e, per contrario, l'aaplrazione all'arte fondata sul vago, sull'indeterminato, sull'ineffabile, che porta alla nostalgia, al dissidio spirituale, al tormento, alla malineonia.

Ed é necessario avvertire quanto sopra per comprendere i succesilvi aviluppi nel campo letterario.

Infatti il primo aspetto porterà al naturalismo, al romanzo speimentale, al verimo, mentre il secondo porterà alle correnti del aimbolismo, del misticismo e del decadentismo, dell'impressionismo.
L'amore della realtà, della storia é poi l'elemento costitutivo ed ispiratore del romanzo storico.

Il sentimentalismo conduce al versamento delle lacrime, agli sdilinquimenti del Romanticismo deteriore, ma, se ben s'indaga, ci si accorge che questi sdilinquimenti, questi pianti, presentano cause molto più vitali. Nasce in questo tempo il mito del dolore con la sua adorazione, come emblema della nobiltà umana.

Natura umana e dolore sono consustanziali. E' questo il sentimento che ci distingue dai bruti. Il dolore é prodotto da varie cause: il romantico ha vivo il senso del mistero, non riesce a prefiggersi mete ben chiare, continuamente (anche se sa che é vano) ricerca una risoluzione, un punto d'appoggio.

$\mathrm{Da}$ qui la caratteristica malattia della volontà che non sa agire perché non sa dove dirigersi. E se questa malattia é propria di ogni spirito romantico di qualsiasi tempo, che però la sente come un difetto, il romantico, diciamo, storico la crede una prova di profondità e di alto sentire. Oltre alla malattia della volontà, l'altra caratteristica dei romantici é il conflitto tra individuo e società, fatto che sta alla base di tanti poemi, drammi e romanzi di questo periodo. (Schiller, Alfieri, Leopardi, Byron, ecc.)

Questo conflitto ha due aspetti: il titanismo e il vittimismo.

Il primo consiste nella rappresentazione di individui che combattono fino all'estremo eroicamente contro tutti e contro tutto, pur sapendo vano cgni loro sforzo; il secondo consiste nella rappresentazione di individui che si cullano nel dolore assaporando con voluttà le proprie sofferenze.

Questi atteggiamenti sono due opposte malattie tipiche della malattia della volontà; titanismo é infatti sinonimo di volontarismo esagerato e vittimismo é sinonimo di abulia, a cui porterà il romanticismo decadente per allontanare da sé "il tarlo del pensiero". Un altro aspetto del Romanticismo é la Sehnsucht, parola che non si puó tradurre, ma che indica la tendenza dell'animo verso arcane felicità, arcani mondi di sogno, mai raggiungibili. Si avverte una eostituzionale scontentezza del presente, la mancanza di un ideale preciso che fissi una meta chiara, definitiva e con voluttà ci si abbandona a questi sentimenti che costituiscono anche uno dei prinelpall aspetti della lirica leopardiana. Dalla Sehnsucht nasce anche la liaba, che ha pure origine nel culto romantico della poesia popo- 
lare. Dalla Sehnsucht nasce soprattutto l'esotismo, il desiderio di trasferirsi in luoghi lontani e sperduti, dove non giunga l'eco delle envenzioni sociali e l'uomo possa vivere puro al contatto della nalura. Questa é anche una risoluzione romantica del conflitto uomoocirth, a cui già abbiamo accennato, conforme all'insegnamento del '700 e del ROUSSEAU in particolare.

La thardino come nell'Arcadia, ma tumulto, tempesta, lotta, pericolo he Incombe sull'uomo, il quale non é più una creatura perfetta, ma centro di deformità e di brutture, specchio del dolore e dell'imperlarlone che sono nelle cose. Dalla scoperta del dolore nasce l'amore per esso, la voluttà del soffrire.

Esposti succintamente i caratteri generali del movimento romantico, sarà più facile vedere nei particolari più segnatamente stoiel le fasi che conducono all'invenzione della forma di romanzo che a nol interessa.

Abbiamo già parlato di Romanticismo perenne e di Romanlieismo storico che certi critici come Vittorio Rossi vorrebbero fare inlziare dal '500.

Anche se non si partecipa questa convinzione, é certo che dalla epoea del Tasso in poi spira aria rivoluzionaria nel campo letteraio, rivoluzione intesa come avversione alle regole prestabilite, alle unita aristoteliche, ecc.

$\mathrm{Cl}$ si oppone al classicismo che era stato in vigore fino a tutto Il periodo rinascimentale. Dopo il Tasso, tanto per rammentarne qualcuno, il Marino, il Tassoni, il Metastasio, il Vico, Carlo Gozzi rappresentano questa tendenza e costituiscono, per cosi dire, un preromanticismo.

Carlo Gozzi é il più vicino pre-romantico, ma i germi del nuovo movimento sono evidenti nel '700 e sempre più infittiscono man mano che ci si avvicina all'800. Hanno inizio gli studi delle letterature atraniere, soprattutto della tedesca, s'infiltra nella letteratura เиล веmpre più penetrante vena di tristezza e di sconforto, sorge la commedia "lacrimosa", viene di moda la poesia sepolcrale e accanio a questa, quella bardita e ossianica. Il Cesarotti appunto, traduttore di Ossian, indica nella malinconia il distintivo del genio. E guesia tendenza divenne moda ed indicò, se non altro, il desiderio il aseire dal vecehi schemi, di liberarsi dalle vechie regole, Prima che il Romanticismo si affermasse definitivamente e vigorosamente, esistono tentativi di conciliazone fra la ragione, tanto esaltata dallo Illuminismo, con il sogno, così amato dai romantici. A questo proposito il Bettinelli scrisse che l'arte é "un sogno che si fa in presenza della ragione". E il Pindemonte: "Giova intanto l'andar sognando, poiché il vegghiare é sì tristo: giova, vivendo con la fantasia in un sistema di cose tutto ideale, ma però riducibile al vero, toglierci cosi a questi oggetti increscevoli che ne circondano e la pessima fortuna nostra per qualche modo ingannare".

Gli uomini alla Pindemonte, pur accogliendo nuovi spiriti e sentimenti, restano fedeli alle forme tradizionali.

"Antica l'arte-onde vibri il tuo stral,

ma non antico sia l'oggetto in cui miri"

Questo precetto del Pindemonte ripreso da Andrea Chenier é il precetto base del neo-classicismo fine secolo imbevuto di spirito romantico che costituirà la caratteristica del Romanticismo italiano, specialmente in ciò che questo movimento ha di più grande: Foscolo, Leopardi, Manzoni.

Non diversa dal Pindemonte é la posizione dell'Alfieri, anche se l'espressione é diversissima ed egli rappresenti il titanismo, il senso della solitudine e l'individualismo romantico.

Però gli eroi alfieriani sono titani che non conoscono l'ineluttabilità del fato e la vanità delle loro epiche azioni; conoscono solo vittorie ad ogni costo e sono padroni della volontà di cui abbiamo parlato più sopra. Inoltre egli rispetta sempre gli schemi classici.

Comunque siamo nel periodo del pre-romanticismo.

Il termine romantico "romantic" appare in Inghilterra verso la metà del 17 secolo con significato spregiativo (come molti altri termini indicanti movimenti letterari nuovi atteggiamenti spirituali e pilehici), ad indicare cioé il carattere puramente fantastico, irreale, caratteristico dei romanzi allora in voga.

Corrispondeva press'a poco a quello che noi oggi intendiamo eon II termine "romanzesco". Tale termine però, accanto a questo alinifieato, fine dal suo primo sorgere, ne ha uno positivo: riferito Alla natura significa pittoresco e indica l'amore per gli aspetti selvaini e malineonici della natura.

1. "romanzeseo" chamerd II Pindemonte quello che altrove si hilama ula declaamente romantico. 
In questo tempo il vocabolo é già in voga in Francia ed indica l' l'a natura, ma anche lo stato d'animo che esso suscita.

A questo punto della sua evoluzione semantica, il vocabolo é maturo per essere considerato in Germania come termine indicatore masce il contrasto fra classico e romanHeo.

Herder si serve della parola romantico per indicare medioevale In contrapposto ad antico, cioé per indicare la poesia sentimentale ed ingenua in antitesi con la poesia tradizionale e dotta.

Madame de Stael si incaricherà di diffondere in tutta Europa nuova poetica con il vocabolo che la indica.

In Italia l'articolo della Stael, come abbiamo visto, apparve ne) 1816

Si cominciano così a diffondere le nuove idee di cui il più equiUlbrato, acuto e sensato codificatore sarà il Manzoni.

Fra tutti gli altri atteggiamenti che già abbiamo elencato (abohrone dell'uso della mitologia, dell'innovazione dei classici, delle (a) dobbiamo fermare la nostra attenzione su yuella del "sentimento della vita reale". Lo stesso Manzoni ammeteva che queste idee circolavano già da tanto tempo nel mondo lelterario, ma che solo allora si presentavano in un sistema, si ponevano criticamente all'attenzione umana.

Come abbiamo detto, il Romanticismo presentava una parte arativa (ripudio della mitologia, delle regole, ecc.) ed una parte ositiva non ben delineata, eccettuato il principio che la letteratura fe vuole essere la sorgente d'un diletto intellettuale nobile e durevole.

II Manzoni ammetteva ciò; ma era imbarazzato quando doveva lefinire questo "vero", non quando però ebbe fatta sua l'esigenza lell'arte popolare che avrebbe interessato un maggior numero di lettori perché derivata dai fatti della vita.

II romanticismo appare al suo sorgere con questa particolare apparenza di ragionevolezza contro l'irragionevolezza del movimen. io ayveraario.

Comunque, da buoni ragionevoli, i romantici non vogliono essere eatremiati e cercano di conciliare le opposte lere tendenze con quelle degli avversari. E Conciliatore si chiamerà appunto il giornale del nuovo movimento letterario.

Vollero conciliare l'antico e il moderno illudendosi che potesse effettuarsi una conciliazione duratura. Essi credono di poter conciliare le regole degli scrittori classici con le esigenze romantiche, affermando che anche i classici hanno sovente e a loro modo trasgredite le norme letterarie e comunque la loro gloria non consiste nell'averle osservate esternamente. Tutti i grandi poeti del passato sono ritenuti romantici e posscno educare i letterati moderni che devono avere una solida educazione letteraria. Con questo spirito si studiano Dante, Petrarca, Tasso e Arosto. Si deve respingere l'imitazione, ma non lo studio dei grandi.

L'arte deve essere rivolta al popolo: per popolo si deve intendere non i troppo civilizzati e neanche $\mathrm{i}$ troppo ignoranti. (Dunque in Italia si era lontani dalla concezione aurorale e istintiva della poesia già in voga presso altri popoli dell'Europa)

Con la ragionevolezza quindi il Romanticismo italiano voleva combattere il razionalismo settecentesco, cioé con le stesse armi, ed é quindi in contrasto con l'arazionalità dell'arte pur rifacendosi dal Vico. Diversi, quindi, giova ripeterlo, dai romantici nordici e germanici, pervasi tutti di misticismo egocentrico, nostalgia ansiosa di arcani mondi, ripudio di ogni congiuntura fra ispirazione ed espressione, sbandamento perplesso.

In Italia i Romantici conservano la quadratura dell'arte che si mantiene ancora solida seguendo il capo, Alessandro Manzoni, ed ammirando come predecessori il Parini e l'Alfieri (sebbene questo ultimo soprattutto da un punto di vista patriottico), senza che ne fosse penetrato il valore di protoromantico che consiste nel suo individualismo incoercibile, nella sua scontentezza del presente e nella sua profonda malinconia, anche se questa ha un carattere tutto particolare. Come si vede quindi, il Romanticismo in Italia assume peculiarità diverse.

Non si deve però credere che il Medioevo con la sua mitologia barbarica e cristiana anche nel Nord fosse la fonte più ricca e cui i letterati attingessero. Ma tuttavia é vero che le due ballate del Burger intitolate Cacciatore feroce e Leonora costituirono il modello della nuova poesia anche in Italia, specialmente per riflesso, nell'opera della Stael e nella Lettera semiseria del Berchet. 
Un nuovo armamentario invase il campo letterario, armamentario che acquistò presto il favore dei lettori, ma che perse il credito oltenuto: da un lato amori puri e infelici e dall'altro streghe, scene Infernali, spettri, ecc.

Siccome lo scrittore deve essere attuale, si ispira al vero e alla vith, parla cioé all'esperienza, trae la materia dei suoi scritti dal Criatlanesimo e dal Medioevo da cui il mondo moderno ha avuto orifine e ha tratto le sue basi. Non più il mondo antico pagano e con mili non più attuali.

Questo in sostanza é un sofisma perché a una mitologia ne venlva sostituita una altra, a regole precedenti altre regole, sebbene II tutto più attuale e moderno.

Il fatto é che gli uomini non possono fare a meno dei miti e nemmeno, però, sanno restare eternamente in quelli già creati o trovati bell'e fatti. Sentono il bisogno di crearne dei nuovi che semҺrano più ragionevoli. Oggi però si é giunti ad un'epoca in cui ogni cosa umana, ogni scoperta, ogni motivo in ogni campo, nasce con la coscienza del suo limite e della sua relatività. La critica, più forse di ogni altra attività umana, dovrebbe assumere questo atteggiamento di fronte agli oggetti da giudicare, inchinandosi di fronte ai grandi di eul cerca di spiegare il significato e di giudicare il valore, ma con plena coscienza del suo limite e del carattere problematico e relaivistico delle sue affermazioni. Ciò in quanto ormai anche le scienze asatte sono conscienti di tale circostanza. La cultura inoltre, intesa come erudizione e conoscenza di fatti e fenomeni, é una convenzione empre sottoposta a cambiamenti in seguito a nuove scoperte e lotiche (nel momento in cui vengono formulate) conclusioni.

Ben presto il popolo non credette né amò gli spettri né la mitologia medioevale più di quanto non amasse né credesse alle divinita pagane. Che fare dunque? Bisognava rivolgersi alla storia, sola verità reale, non discutibile e accettata da tutti. Qui comincia l'argomento specifico del nostro tema.

Era tuttavia necessário, come é facile comprendere, esporre in qual modo si giunse a questo punto.

La fedeltà all'attualità portava al realismo; ma la realtà era ea te del teatro, della commedia e dell'invettiva. Oceorreva una coneiliazlene fra l'esigenza realistica e la tradiziene, Biaesnava inoltro conciliarla con la fiaba, con il sogno, la Senhsucht, di cui abbiamo più sopra parlato. Conciliazioni difficili perché poste fra termini contraddittori. Da ciò la lirica storica, la novella in versi e il romanzo storico che i primi romantici prediligono.

Certamente il Manzoni, grande artista, portò in un modo tutto suo, originalissimo e grande, questa esigenza realistica e questa conciliazione di realtà e di fantasia nel campo letterario. La fusione delle opposte tendenze é cosi alta, naturale e grande che quasi non si scorge tanto é unificata da una sublime omogeneità di ispirazione.

Nei minori e nei minimi, tutlavia, le due predette esigenze le avvertiamo come sovrapposte. Dice U. Bosco (op. cit. pag. 628): "La quotidianità dei casi di Renzo e Lucia, umili che per la prima volta nella storia della letteratura italiana entrano romanticamente nel regno dell'alta poesia, é riscattata dalla nobiltà dell'assunto storico-educativo che essi sono chiamati a servire; la stessa lontananza nel tempo contribuisce ad eliminare il troppo quotidiano, sempre sgradevole a occhi italiani. Per mezzo della storia, la realtà é per così dire aulicizzata, e perciò acquista diritto di cittadinanza letteraria".

Questo é il punto fondamentale da ben meditare per comprendere le esigenze spirituali ed estetiche da cui trasse origine il romanzo storico.

II Thierry aveva compreso l'importanza del nuovo genere e cio che il Manzoni avrebbe potuto realizzare. Dice Angelo $\mathrm{De} \mathrm{Gu}$ bematis nella raccolta di lettere manzoniane da lui fatta: "Io non ho bisogno di rilevare l'importanza di un tale giudizio proferito dal Thierry, cioé dal vero fondatore della nuova scuola storica in Franela, da colui che mostrò poi con tanta verità ed eloquenza che la atoria, bene investigata da prima e poi animata dal soffio dell'arte di chi viene a narrarla, é il più ricco e il più bello fra tutti i romanzi (II Therry aveva scritto in una lettera al Fauriel:-Il faut absolument que Manzoni vous parle comme moi là dessus. C'est lui qui est maintenant votre conseiller intime et votre compagnon de tous les

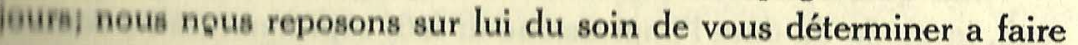
e que vous serait à la fois le plus honorable et le plus utile. Dites lit de ma parte que je désire singulierèment voir son ouvrage pour time décider sur la question du roman historique et peut étre essayer hat mêtme quelque eomposition de ce genre. Je ne sais pourquol je 

m'imagine que autre ami pourrait bien fonder en France une école nouvelle de romancier et onner du courage à ceux qui n'osent point encore traiter ce genre de peur de copier Walter Scott.

Come l'aspettativa gloriosa del Thierry doveva rispondere al to desiderio, all'intendimento artistico continuo ed intenso del Manzoni!

Come questi, nel leggere il passo della lettera che il suo caro Cott'occhi, senza dubbio, dovesse sentirsi dilatare a nuova potenza il genio che si trovava un'altra volta compreso!

Quanto viva ora la compiacenza nostra nell'udire un Thierry augurarsi già che il Manzoni nostro regali alla Francia com suo capolavoro, l'esempio di una nuova letteratura! $\mathrm{E}$ come dobbiamo, ora purtroppo, sentirci meschini noi, che, scrivendo, pensiamo così poco ll'arte nostra, ed agli effetti buoni o tristi che la nostra parola parata o scritta dovrebbe, se fosse ancora sincera e potente, produrre in chi la legge e in chi l'ascolta!

Tommaso Grossi é l'esponente più cospicuo della novella in t'argomento storico e l'lldegonda otterrà un famoso successo altestato anche dal Manzoni il quale nel 1823 concludeva una sua lettera al D'Azeglio dichiarando che il Romanticismo inteso come "non so qual guazzabuglio di streghe, di spettri, un disordine sistematico, una ricerca stravagante, un'abiura in termini del senso comune, era ormai morto, anzi mai esistito".

Racconto e novella storica, in prosa e in versi, sono quindi la prima tappa del nostro Romanticismo in cui per un po' di tempo a a appaga e che preludono al romanzo storico vero e proprio.

Tappa, non meta finale, questa, anche se per un momento Terché conciliava la realtà con la tendenza dell'anima âmantica al sogno e all'evasione di quella stessa realtà.

Per questo motivo l'lldegonda di Tommaso Grossi ebbe tanto ucesso. "Le fantastique du réel de la vie" sembrava aver trovato immagine in essa.

Ma questo concetto, di trattare cioé argomenti di ogni giomo, reall, in modo da dare l'impressione di fatti soprannaturali e romanliel вi trova gia agli inizi del Romanticismo in Inghilterra nel Wordsworth e nel Coleridge.

\section{ORIGINE E CARATTERI DEL ROMANZO STORICO IN ITALIA}

Pure dall'Inghilterra provenne il modello del romanzo storico e Walter Scott ne fu il maestro. Egli insegnò il segreto di ispirarsi, ad un tempo, alla realtà e alla fantasia. E questa fu la causa del suo sucesso in Europa, piuttosto che le sue qualità d'artista non certamente grandissimo.

Egli piacque ai nostri ragionevoli romantici perché ha la sua base nel settecento raziocinante e perché s'ispira alla storia restandole fedele, nella cui trama inoltre sa intessere verosimili fatti fantastici.

La storia da lui considerata é quella di avvenimenti piuttosto lontani, che può essere trattata liberamente, senza preoccupazioni di giudizi troppo recenti o contemporanei e che già di per se stessa abbia un carattere di fiaba affascinante. E' questo un atteggiamento tipico di ogni scrittore di romanzi storici di questo periodo, sebbene ognuno, naturalmente, abbia particolari tendenze e preferenze. Il Manzoni é pure su questa via, benché egli, come accade per ogni grande, anche se appartiene ad un determinato movimento o ad una determinata scuola, deve considerarsi a sé.

Ben presto questa fase letteraria romantica di storico-fantastico generò sazietà, anche perché produsse opere in sostanza piuttosto mediocri e perchè la storia era ormai divenuta un pretesto per colorire il fantastico e la let'eratura fu pervasa da una nuova e recente mitologia (streghe, spettri, amori infelici, ecc.)

Un'altra caratteristica di quesia letteratura sia essa ispirata o meno alla storia é l'umorismo, di cui si servì anche Walter Scott. Interessante é poi vedere come, ad esempio, nel Grossi, coesista questo umorismo con l'altro atteggiamento lacrimoso e sentimentale e come anche il Manzoni se ne serva nel suo capolavoro portandovi una nota realistica (Don Abbondio, Donna Prassede, Perpetua, ecc.) Questo atteggiamento perdurerà, come tradizionale, a testimonianza del realismo romantico e dei suoi frutti, anche negli scrittori succesivi come il Guerrazzi, il D’Azeglio, il Nievo, il Verga, il Fogazzaro e Pirandello.

La tendenza realistica portava i romantici ad auspicare un rimnovamento della lingua che meglio si adattasse ai nuovi orientamenti letterari, problema che tenne molto preoccupato anche it 
Manzoni, il quale sentì l'insufficienza della lingua letteraria tradisionale codificata dalla Crusca. Bisognava, oltre tutto, abolire anhe quest'altra regola che imponeva nel linguaggio letterario di seuuire modelli e codici. Quindi cominciò il nuovo movimento per adeguare il linguaggio all'attualità dei fatti e la lingua scritta alla lingua parlata, sfruttando anche ciò che $\mathrm{i}$ dialetti potevano apportare di buono e di vivo. Esigenza, questa, sentita per tutto l' 800 . Vero $b$ che permarrà molto radicata la convinzione di una lingua aulica e tradizionale ed anche gli stessi romantici (Manzoni comвгево) saranno alquanto illusi sulla loro innovazione. Infatti, perfino I Manzoni, quando crede di avere risolto il problema prendendo come modello la "lingua parlata dei fiorentini colti", concepisce popolo come il terzo stato. Dice il Migliorini a questo proposito: "Ma II romanzo raggiunse ugualmente lo scopo che il Manzoni si proponeva: di raccostare lo scritto al parlato, di dare un colpo moriale al fronzoli retorici che per secoli avevano aduggiato la letteralira Italiana (op. cit. pag. 58)"

Come si vede anche nella questione della lingua erano evidenti le aue convinzioni letterarie di rendere l'opera d'arte aderente alla vita, intelligibile al popolo, staccata da ciò che era tradizionale e morto.

Dice ancora il Migliorini a proposito del Manzoni: "Il suo penalero in fatto di lingua si viene mutando da "federalista" a "unitaกิ०" | spinto dal bisogno di trovare un saldo ubi consistam, sociale a non solo letterario, egli abbandona la primitiva opinione che biตognasse attingere ai tratti simili fra $\mathrm{i}$ vari dialetti per giungere a una lingua comune, e piega sempre più verso l'uso toscano. Qualche ละถะ ancora e poi, meditando sulle varietà che si presentano nello ambito dell'uso toscano restringerà ulteriormente il suo canone, e parlera soltanto di fiorentino" (ap. cit. 55).

Alessandro Manzoni nel discorso intitolato "Del Romanzo stodeo" chiarisce l'ibrido compromesso da cui questa specie di roman30 era sorto. Confessa anche di credere di essere avanzato sulla atrada del Romanticismo, ma di essersi contemporaneamente mantensto coerente al movimento stesso.

Avvertiva anche lui, pubblicando il discorso, che le sue nuove alfermazloni non andavano d'accordo con quanto aveva affermato nella lettera allo Chauvet (1). Però, dice, che se ha cambiato parere non é stato per tornare indietro. Se veramente abbia progredito non sa, e di ciò giudicherà il lettore.

Il Manzcni aveva risposto acutamente al suo contraddittore (Chauvet) che "l'essenza della poesia non consiste nell'inventare dei fatti: questa invenzione é ciò che può esserci di più facile e di più volgare nel lavoro dello spirito, ciò che esige meno riflessione e perfino il meno di immaginazione". "Compito del poeta é l'interpretazione della storia, il completamento per restituirne, per così dire, la parte perduta. Il suo scopo é di raggiungere il vero e perciò più il poeta sarà aderente alla storia come egli l'ha conosciuta $o$ come egli l'ha ricostruita e più otterrà il suo proposito.

Può darsi che lo scrittore scopra egli stesso fatti nuovi, quindi noti a lui solo e che perciò non gli verrebbero rimproverati se egli li tacesse.

Ma perché? Se egli ha scoperto qualcosa di reale, se ne deve servire per arrivare al vero. Dunque la storia é rcaltà e la poesia nasce sclo da questa: "Les faits par cela même qu'ils sont conformes à la vérité pour ainsi dire materielle, ont au plus haut degré, le caractère de vérité poétique que l'on cherche dans la tragédie". Come già si é detto, li Manzoni poi, nel Discorso sul Romanzo storico afferma di aver fatto un passo avanti a queste teorie negandole e ponendo come epigrafe al suo discorso un detto di Cicerone, da cui scaturisce il succo del contenuto di esso: "Intellego te, frater, alias in Historia leges observandas putare, alias in poemate". Avverte insomma che il romanzo storico dopo trenta anni di moda é ormai morto, nonostante la bellezza del modello da lui stesso fornito; e comprende che se il genere ha prodotto cose artistiche vitall, le ha prodotte in quanto opera d'arte, romanzo, invenzione, poesia, non storia. Resta tuttavia valido per lui il principio che ogfelto della poesia sia il vero, ma un vero diverso da quello storico, il verosimile, come egli dice. Verosimile significa diverso dal "certo" (Vico), materiale d'arte manifestato e appreso come verosimile, diverao dal reale "ma un vero veduto dalla mente per sempre o, per parlar con più precisione, irrevocabilmente".

Con cì ai vuol significare che il certo di oggi può essere di-

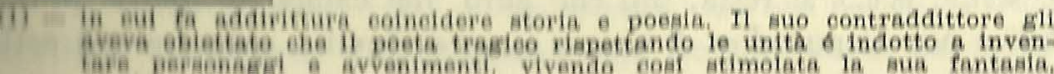

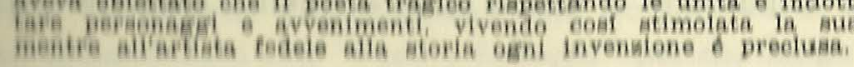


atrutto dal certo di domani, ma il vero poetico é sempre immutabilmente quello che é e l'assentimento che il lettore dà al vero storico f diverso da quello che dà al vero poetico. Da ciò consegue che II Manzoni fa sua la distinzione vichiana fra l'attività logica che il svolge nella storia, dall'attività poetica. Tutte e due non possono coesistire in una sola opera d'arte, la cui natura é appunto essenvalmente arazionale. Così il processo del Romanticismo é compiuto anche nel "classico Manzoni". Se dunque il romanzo storico in delinitiva é valido soltanto perché é invenzione, una varietà del "romanzo" da cui ha preso il nome e di cui é una nuova forma, ne deriva che é pienamente valido anche il romanzo d'argomento contemporaneo tutto inventato. "Voglio dire il romanzo nel quale a) fingono azioni contemporanee: opera affatto poetica poiché, in inisi, e fatti e d'scorsi, tutto é meramente verisimile".

Verosimile nel senso detto più su.

Non giungerà, tuttavia, alla piena legittimazione di esso per un resto di pregiudizio tradizionale. Infatti alle parole più sopra Aportate egli aggiunge:

"Poetica però, intendiamoci, di quella povera poesia che può uacire dal verosimile di fatti e costumi privati e moderni".

In Francia questo preguidizio era stato già superato dal Balvae,

Bisogna poi osservare che la storia del Manzoni non é quella vera (poiché non esiste una storia vera in senso assoluto uguale per (utti), ma quella che a lui pare tale, sempre per quel relativiamo e quella impenetrabilità dell'io di cui spesso si é parlato nel nostri scritti. Inoltre influ'scono sull'interpretazione storica fatti eontingenti come l'erudizione limitata o troppo ampia sopra l'argemento preso in esame, la qualità dell'ingegno, le tendenze politiche, ecc. - Lo stesso Manzoni confessò di essersi sbagliato riguardo all'Adelchi. Oltre quindi al relativismo universale, a cui ioggiacciono necessariamente tutte le attività umane, esiste un relaliviamo individuale dovuto a tutte le varie contingenze ed alle pecullarita degli individui.

Vero nella storia é solo il fatto esterno, documentato e testimoniato in vario modo, ma per il resto (cause intime, intenzioni e bili e non al puó dire di essere sempre giuntl a separare con certa acienza.
La teoria del romanzo storico, quindi, da un punto di vista rigorosamente razionale e scientifico, é una illusione perché si pretende di ricostruire, secondo le testimonianze storiche, la psicologia e la vita di personaggi esistiti o che avrebbero potuto esistere come attori di determinati o finti avvenimenti. E' un'illusione anche per questi motivi: le testimonianze storiche sono fredde enunciazioni di fatti esterni e il romanzo storico pretende dar intima vita ad essi completando in certo senso la storia. Ma si possono conoscere mai le vere e segrete cause, specialmente nelle loro connessioni e finalità, degli avvenimenti, tanto più se questi sono di natura psicologica? Si può mai conoscere con certezza da quali peculiari moventi psichici un uomo sia stato spinto ad una determinata azione? Non gioverebbe forse allo scopo, anche potendolo, nemmeno una confessione fatta di viva voce. Il romanzo storico non può pretendere di essere più vero della storia, di essere storia vivificata; non si può dar vita alla storia se di ques'a, come abbiamo detto, ci si deve accontentare di conoscere il solo fatto esterno. E' quindi anch'esso un'opera di fantasia, di immaginazione e come tale può essere vera opera d'arte. E non é da credersi che non possa rispecchiare i tempi e i personaggi che vuol rappresentare: li può rispecchiare, dare un'idea, ma non pretendere di ricostruire la realtà che fu o che avrebbe potuto essere, anche perché i fatti tramandati sono stati filtrati attraverso l'interpretazione degli uomini, oltre che dalla personalità dello scrittore. Ricostruire la verità storica in senso assoluto é cosa impossibile, non invece ricostruirla in senso relativo o della verosimiglianza. Il romanzo storico é quindi, a suo modo, verista, specialmente quando lo scrittore cerca di evitare ogni esprit romanesque.

L'autore che si cimenta in questo genere letterario e che scrive rispettando con il massimo scrupolo la teoria agisce con la stessa paleologia degli attori teatrali quando interpretano con serietà ed impegno un testo a cui cercano di essere fedeli il più possibile. Lo acriltore di romanzo storico cerca di interpretare i fatti e i personaggi artisicamente rispettando si le fonti e le sue convinzioni storiche, tha anche sbrigliando il proprio estro creativo.

II Tommaseo nel 1847 si era attenuto ancora nel suo "Duca il Atene" al modello atoricheggiante, ma tre anni dopo, nel 1840 , Faveva abbandonato eon "Fede e belleza" per dar luogo allo stu- 
dio psicologico, al fatto privato o contemporaneo al posto della revocazione e della illusoria ricostruzione storica di fatti e personaggi lontani.

Anche il Nievo si porrà su questra strada. Ma a segnare la nuova via del Romanticismo, vale molto più dell'opera del Tommaneo, un'operetta assai minore per valore artistico ma di grande riaonanza a quei tempi: l'Edmenegarda di Giovanni Prati. Il poemetto prende infatti le mosse da un argomento di cronaca giornaliera: l'adulterio di Edgarde, sorella di Daniele Manin, eroina vivente di una comune storia narrata con particolari altrettanto comuni. Appunto per questi nuovi aspetti il poemetto fu applaudito. II precedente romanticismo, di cui Manzoni era stato l'esponente, nembrava ormai troppo "ragionevole", troppo poco mistico ed egocentrico, troppo poco romantico.

La folla assetata di questi ideali poetici e che fino ad allora Ii aveva cercati in poeti stranieri come il Byron, il De Musset e il Lamartine, ora crede di averli trovati in un poeta italiano. Il mito romantico del conflitto individuo-società é alla base del poemetto. L'adultera non é compresa dagli uomini né dalle leggi; e nemmeno il aeduttore. Entrambi sono vittime della fatalità. Il motivo del vittimiamo trova così una nuova espressione, vittimismo che prevale nella letteratura. Questo atteggiamento fa ritenere tutti vittime, tutil incolpevoli e cerca solo di spillare lacrime quante più può. Il poemetto Edmenegarda del Prati deriva dalla Parisina di Byron ma fiaente anche di motivi alla Chateaubriand e alla Lamartine. L'atlesgiamento lacrimoso non era stato certo del Manzoni e provocherà le ire del Carducci e degli scapigliati ultraromantici. L'altra novitá del poemetto, che pure tuttavia é una nuova prova dello ideale romantico di conciliare la realtà con il sogno, consiste nello atudio psicologico "oggettivo" dei personaggi, studio che le narrarioni in versi avevano fino ad allora impedito.

La realtà oggettiva, a cui pure lo scrittore deve ispirarsi, é povera di spunti poetici, ma da essa si deve estrarre il fantastico. Da questo lavoro si acquista coscienza della propria anima e della altrui, del travaglio per cui essa si nobilitò sentendo, comprendendo e soprattutto soffrendo.

Cosi ef al aente vittime, ci si lamenta, ef al autcommisera. Da elo una perpetua scontentezza, una volutié di planto consola- tore. Aleardi e Prati tuttavia non sanno rendere nitide le immagini del loro mondo e neppure sanno rendere efficacemente la poesia del vago. Le loro immagini sono sfocate e se la loro poesia tende alla musica, anche questa resta esterna, al di qua molto spesso della poesia. Però in questi due poeti si trovano germi efficaci per sviluppi futuri. Non é dunque da sottovalutare la loro opera. La tendenza a superare lo sfocato, il sentimentale e lo sdilinquito, si fa manifesta negli avversari e così si hanno le diverse tendenze letterarie:
1) la scapigliatura
2) il verismo
3) il carduccianesimo

Il primo movimento é quello dei poeti che negano alla poesia ogni finalismo, affermando la completa autonomia dell'arte. La poesia é immediatezza d'espressione, senza preoccupazioni stilistiche: una stenografia. Tutte le arti sono identiche tra loro ed hanno per unico scopo di esprimere con vari mezzi tecnici (parola, nota, colore, ecc.) la poesia dell'intimo. La scapiglitura si può considerare il terzo romanticismo.

Gli scapigliati vollero reagire al pratismo e all'aleudismo, allo sfocato nella arte con l'individualismo, il nuovo, l'oiginale ad ogni costo. Dopo di essi nascono la letteratura realistica e familiare o borghese o veristica e il realismo carducciano.

Abbiamo voluto schematicamente tracciare $i$ vari aspetti del Romanticismo, epoca in cui sorge il romanzo storico, e quelli dello 800 in genere con i germi anche della letteratura del novecento, in quanto, sia pure transformato in confronto alla primitiva origine, il romanzo storico ha conservato la sua esistenza fino ad oggi (Il Molino del Po di Bacchelli) adattandosi naturalmente di volta in volta alle nuove tendenze letterarie.

Con gli scapigliati tuttavia ci si incammina verso il concreto, esigenza somma del Carducci e quanto mai romantica, anche se il poeta credeva di essere un anti-romantico dichiarato.

A causa di questo atteggiamento é naturale che egli si rivolga alla storia da lui nobilitata però con l'ala degli anni trascorsi sui fatti e I personaggi descritti; atteggiamento in tutto simile all'eaperienza dei primi romantici a cui il Carducci aveva voluto anche reagire, E per concretizzare la storia, egli la considera come realtà 
in atto immergendola soprattutto nel paresaggio, testimone vivente del fatti antichi.

La storia poi, da nozione libresca, in lui diventa vita, forma, alta. Basta pensare alla realtà scultorea dei suoi perscnaggi.

E' appunto per questa sua nobile qualità di scultore che in lai 11 reale non é più borghese e viene allontanato dal contingente.

$$
-\mathrm{OO}-
$$

Come si sa, il nostro Rinascimento ebbe un grande influsso ulla letteratura inglese, la quale non influì mai ugualmente su quella italiana all'infuori del periodo romantico e precisamente com W. Scott e Byron.

I vantaggi che ne derivarono all'Italia furono ben scarsi, perhé se si esclude i Promessi Sposi di A. Manzoni che, del resto, ha pregi tutti particolari quale altra opera di autentico valore possiamo trovare fra gli imitatori dei due grandi inglesi?

II primo ottenne in Italia e in Europa in genere un'ammirafone molto superiore a quella ottenuta in Inghilterra, dove il viatoso scrittore influi soltanto sugli autori di minore importanza. Lo Scott infatti, sebbene avesse avuto in Inghilterra qualche predeeеввог, seppe creare l'illusione del passato, seppe narrare una belî storia romantica senza oltrepassare mai i limiti della probabilita, in una cornice veramente storica che faceva rivivere il pasato nella realtà del presente. E la sua pittura é spesso viva ed amozionante si da essere imitata da famosi scrittori come Dumas, Huge, Manzoni.

In Italia, ammirato anche per la sua opera poltica oltre che per quella letteraria, ebbe schiere di traduttori, fra cui il Pellico, di imitatori fra cui il Guerrazzi. Amato contemporaneamente dai emanticl che inneggiavano a lui come a un maestro e dai classici come il Monti, il quale affermava che il suo Romanticismo era di un genere cosi sublime che Omero stesso gli avrebbe perdonato. Che altro dire poi della fortuna di W. Scott. in Italia?

II suo primo romanzo tradotto in italiano fu Kenilworth (1821, Ferrario), a cui seguirono, dato il precedente successo, I'lvanhoe (1822) legend of Montrose (1822), Le prigioni di Edimburge (1823), l'Antiquario, ecc.

Nello atesso anno della versione ftallana del auo primo romanzo vennero in luee due traduzioni del poema The lady if the
Lake a Torino e a Palermo le quali destarono nella critica più echi dei romanzi, che però ebbero tra il pubblico molto seguito e consenso; soprattutto a Milano dove si ebbe un'immensità di traduttori e di editori. In Italia, poi, nella prima metà del sec. 1800 si può dire che non vi fu editore che non pubblicasse qualcosa dello Scott. A Firenze, che seguì ben presto l'esempio di Milano nel pubblicare lo Scott, si pensò perfino a una collana di romanzi storici originali italiani (1830 Editore Veroli).

Ai romanzi scottiani si ispirarono artisti e musicisti come Rossini ne La donna del lago, il Pacini con l'lvanhoe, Donizzetti con la Lucia e l'Elisabetta al castello di Kenilworth.

Enorme fu il suo influsso: il romanzo storico da lui derivante e da lui imitato fu in voga dal 1826 in cui apparve il Castello di Trezzo del Bazzoni fino al 1860 circa, tanto per delimitare schematicamente dei termini cronologici, perché influssi si ebbero anche dopo, fino a tempi recentissimi. Fra i più noti imitatori, oltre il Bazzoni: (1) Il Manzoni (sebbene questi superasse il modello con una personalità tutta sua della quale parleremo in particolare), il Grossi, il D'Azeglio, il Cantù il Varese, il Rosini e il Guerrazzi.

Prima degli anni 1826-27 non si hanno, in generale, vere e proprie imitazioni scottiane. La via non era facile né libera. La vera e propria imitazione scottiana comincia con gli scrittori sopra menzionati ed inoltre il Lancetti, lo Zorzi, il Falconetti e qualche altro. Il Varese é tuttavia il massimo tributario e il massimo esemplificatore dello Scott (La Sibilla Odoleta). Spesso nella letteratura italiana però ci si era ispirati alla stcria e già l'Innamorato del Boiardo può essere benissimo considerato un romanzo storico, per cosi dire, scottiano tanto sono gli elementi esterni che lo avvicinano al romanzo dello Scott.

Il romanzo storico comincia cavalleresco con il Guerrin Meachino, I Reali di Francia, l'Aspromonte. Prossegue con il Boiardo, II Pulei, l'Ariosto; ma in Italia divenne in seguito patriottico e servì a acopl di battaglia politica.

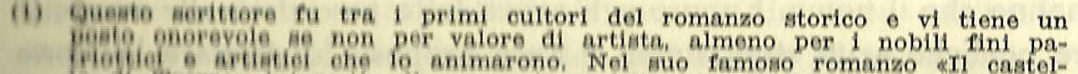

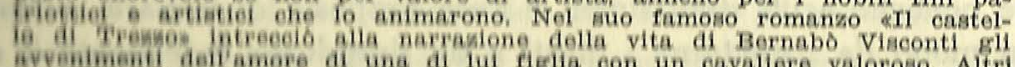

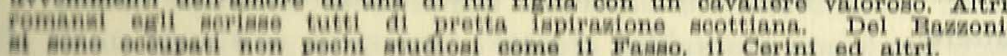


La vita romanzata é una rappresentazione intorno al caratte(6) di un dato personaggio, una ampliazione fantastica, ma verosithe approfondita, della sua vita. Il falso, per gli scrittori di vite omanzate, non cons'sterebbe nel creare avventure non mai comlute, ma nel non saperle intonare al carattere del personaggio di cut si narra la vita. Il romanzo storico architetlava e fantasticava di un fondo di verità che era semplice scenario e spesso era curiosità di rivivere antichi costumi, nostalgie di epoche sorpasate. II Manzoni per questi componimenti misti di fantasia e di atoria impostava rigidamente il problema morale. E non fu male herate venisse l'avvertimento anche per l'opera d'arte ci sia un limite all'alterazione e alla menzogna.

Il romanzo che soprattutto interessa alla nostra indagine stoE la Calata degli Ungheri in Italia nel Novecento (nel Ricolitore, 1822 , ripubblicato a parte nel 1823 e ristampato nel 30 ) he il Bertolotti non solo intitola romanzo storico, ma si dá anthe la pena di forn'rlo di un elenco nelle opere consultate durante 1. eomposizione Qussto romanzo rappresenta il prims tentativo In Italia di imitazione di W. Scott, sebbene com risultati assai diseutibili, Molti, mentre attendevano a seri studi storici, ecc, ne ricavavano nello stesso tempo materia per qualche romanzo, sempre tratti ad alterare la verità pur restando nel verosimile ad accordando ai fatti del vero i sentimenti che da questo vero scaturiscono.

II poeta può interpretare lo stato d'animo d'un eroe in una lata situazione; una parola storicamente esalta può essere svilupta ed ampliata da un certo numero di considerazioni. Una veitá non si presenta mai allo stesso modo a due persone, non é umanamente possibile parlare di obiettività storica. Uno erittore non ci può rappresentare la verità in una maniera impeccabilmente esatta. L'atto stesso del ripensare la storia la rende da osgettiva, soggettiva. Il Manzoni sapeva bene che il romanzo stoteo era un'arma pericolosa ed egli stesso aveva creato dei perscnasid come Adelchi e il Cardinale Federico che di storico non anne il nome. Eppure egli aveva fatto precedere alle sue opere poetiche acuti studi storici e s'era proposto di trattare la storia n modo da rispettame $\mathrm{i}$ diritti e da riprodurne fedelmente il ca rattere.
Il Manzoni, oltre l'esperienza personale, aveva l'esempio dello pseudo romanzo storico italiano patriottico che torceva la storia a fini pratici, in cui la verità si deformava sotto lo spirito di un'animosa idealità e diventava spesso mitologia.

Giacché un'obbiettività assoluta é impossibile, ci dovrá almeno essere l'incorrotta coscienza di chi scrive, il quale non abbia tesi predisposte a cui torcere $\mathrm{i}$ fatti storici, né odii ed amori che gli impediscano di raggiungere quella verità che sola é possibile conseguire relativamente.

In un'opera d'arte tuttavia c’ò che si pretende é l'arte: la realtà dell'arte: la realtà dell'arte é tutt'altra cosa dalla realtà storica. Dice Lopez Celly nella sua opera dedicata al romanzo storico:

"Quindi più che la verità storica, é l'arte che al romanzo storico e generi affini abbisogna. $\grave{E}$, insieme all'arte, come indispendabile elemento estetico e normale, l'onestà. Di questa non si può fare assolutamente a meno".

Abbiamo già detto che il romanzo storico diviene ben presto in Italia strumento di battaglia politica e patriottica. Si ricorderà a questo prcposito l'opera del D'Azeglio che rientra completamente nella sua opera di uomo politico.

I suoi romanzi sono stati costruiti in una sottospecie di clima artistico, cioé entro le precccupazioni politiche. Letteratura di battaglia insomma.

La patria é sentita da un punto di vista puramente pratico. E sono precisamente i problemi pratici che lo spingono a scrivere. Per cui, nei suoi romanzi, l'Italia non é idealizzata, ma discussa.

Romanzi patriottici scrissero anche G. Ruffini, Luigi Capranica e innumerevoli altri imitatori manzoniani. Il prototipo di tutti gli scrittori di battaglia fu F. Guerrazzi, il quale dimostra un amore di patria aggressivo e una passione di partitante all'antica maniera fiorentina.

Anche "Piccolo mondo antico" di Fogazzaro é un romanzo atorico: romanzo anche psicologico che si svolge su uno sfondo Fisidamente storico e ncnostante le vicende del fatto spirituali e private, si respira l'aria drammatica del nostro Risorgimento.

Questo romanzo storico più modemo si differenzierà molto dalla forma primliva, in quanto l'intento dell'autore non é più quel- 
lo di far rivivere la storia (tanto più che il periodo storico trattato palpitante, attuale). Interessa invece all'autore più che altro I latto privato, particolare, quello che ha colpito la sua fantasia (nel caso di Piccolo mondo antico il conflitto psicologico fra marito moglie) sia pure inquadrato nel movimento storico risorgimentale così vivamente sentito e rappresentato. Cosí lentamente il romanzo storico si evolve dalla primitiva forma.

Quando la mcda per il Medioevo si fu alquanto sfogata, il romanzo storico atrinse anche dalla storia romana; ma la storia anche in queste opere d'arte é più o meno fatalmente tradita.

Anche scrivendo una lettera ad un amico siamo portati a prendere un atteggiamento che non é più nostro, non é più veramente sincero.

Lo scenario storico un po' sempre si muove e si transforma atto l'impulso di chi crea, specialmente quando, come nel tempo da nol considerato, l'autore é spinto da caldi sentimenti patriottici aceanto agli abbandoni fantastici e sentimentali.

Ben presto da noi infatti il romanzo storico divenne strumento di propaganda civile e di incitamento alla lotta contro i tranni interni e stranieri.

Dalla Margherita Pusterla di Cantù (1838) dai romanzi D'Azeglio, del Guerrazzi, del Grossi, fino al Nievo, e al Rovan) II genere si rinnova con l'apporto di un materia più varia e più nuova.

Dopo il 27, il genere risente, oltre che dello Scott, dell'influenza manzoniana, sopratutto riguardo alla lingua e allo stile. II piü cospicuo rappresentante di questa influenza é Tommaso Cressi, autore del poema eroico "I Lombardi alla prima Crociata" e del Marco Visconti (1834), storia d'amore ambientata nella Lombardia medioevale.

II Grossi segue la falsariga manzoniana perfino nelle situazioni (iguistici e la sintassi.

PIi che nel Grossi, l'intento patriottico si trova nell'Ettore fleramosea (1833) e nel Nicolò dei Lapi (1841) di M. D'AzeGlio,

L'atmostera di questi romanzi e pii vibrante del tempo. nen maneano gli abbandoni al sentimentainmo
Inolire II D'Areglio al dimostra artista di elfieacla lmpreasionistica e capace di rendere con grande evidenza e con una sola pennellata paesaggi e ambienti.

Altra personalità di rilievo é quella di F. D. Guerrazzi (18041873), rappresentante della corrente democratica del Risorgimentoe uno dei protagonisti della rivoluzione toscana del 48 . I suoi romanzi e racconti fra cui La battaglia di Benevento, l'Assedio di Firenze, la Beatrice Cenci, ecc sono tuttavia da considerare come una battagliera affermazione di sentimenti e di ideali politici piuttosto che opere d'arte.

Nel suo amore per il truce e il violento, per l'orrendo e per il macabro, si manifestano più che in qualsiasi altro scrittore certi aspetti estremi del Romanticismo europeo da Byron a V. Hugo.

Lo Scott e il Byron furcno dunque due scrittori molto in voga nell'Italia e di cui troviamo molte tracce nella nostra letteratura.

Non così dello Shelley del quale invece non vi sono tracce se si esclude il Niccolini e il Guerrazzi e pochi altri.

fale arlige terd conthuaçồ ne próximo número. 